\title{
New Technique for Suppression Four-Wave Mixing Effects in SAC-OCDMA Networks
}

\author{
Ibrahim Fadhil Radhi, S. A. Aljunid, Hilal A. Fadhil, and Thanaa Hussein Abd \\ School of Computer and Communication Engineering, University Malaysia Perlis (UniMAP) \\ Perlis, MALAYSIA
}

\begin{abstract}
A new technique invented for suppressing the FWM in SAC-OCDMA systems based on adding idle code at the sideband of the code construction to generate the virtual FWM power at the sideband of the signal, and then by subtracting these virtual FWM power from the original FWM power in the system and filtering the data part at the channel. This technique is applied for both SAC codes, Random Diagonal Code (RD) and Multi Diagonal Code (MD). Moreover, in terms of cost, the reported technique is considered a cost-effective as the LED light source is used to generate the sideband codes. The results showed that the FWM reduced approximately $25 \mathrm{dBm}$ after using the technique. For example, in the RD code the FWM power at $40 \mathrm{~km}$ fiber length and input power is $15 \mathrm{dBm}$ using the SMF fiber type is approximately $\mathbf{- 5 5} \mathrm{dBm}$ before using the technique, after using the technique at the same values of parameters the FWM power is approximately $-90 \mathrm{dBm}$. In other words, at the MD code the FWM power before using the technique is approximately -61 $\mathrm{dBm}$, the same parameters values, however after using our technique the value of the FWM power is approximately $-81 \mathrm{dBm}$. However, These results gave impact on the Bit Error Rate (BER) also, for example the value of BER in the RD code at the input power $-10 \mathrm{dBm}$ and $35 \mathrm{~km}$ fiber length before using the technique is $1.6 \times 10-23$ and after using the technique the value of BER will become $4.05 \times 10-28$. In addition at the MD code the BER value before using the technique is $9.4 \times 10-22$ and after using the technique the value of $B E R$ is $7.4 \times 10-31$.
\end{abstract}

Keywords-Optical code division multiple access (OCDMA); Spectral amplitude coding (SAC); Multi diagonal (MD); Random Diagonal (RD); Four-Wave Mixing (FWM); Light Emitting Diode (LED).)

\section{INTRODUCTION}

OCDMA is a technique in which user uses a specific unique code rather a specific wavelength or a time slot. The OCDMA uses the spread spectrum technique of CDMA combined with the optical link for transmission of data [1]. The OCDMA provides the large communication bandwidth along with the capability of secure data transmission. The key advantage of OCDMA is the multiple access technique which allows many users to share the same optical link simultaneously.

This is done by giving each user a specific code which can be decoded only by the required user. OCDMA has many unique features that make it favorable data transmissions. Its characteristics make it suitable to increase the capacity and number of users in bursty networks [2]. The OCDMA can accommodate a large no. of channels on a single carrier frequency. It can utilize the bandwidth effectively through coding system. The OCDMA systems provide high degree of scalability and security. It provides high noise tolerance [3].Fiber nonlinearities represent the fundamental limiting on the amount of data that can be transmitted on optical fiber. Nonlinearities of optical fiber can be divided into two categories. The first category encompasses the nonlinear inelastic scattering processes. These are Stimulated Brillouim Scattering (SBS) and Stimulated Raman Scattering (SRS) [4]. The second category of nonlinear effects arises from intensitydependent variations in the refractive index in optical fiber. This produces effects such as Self- Phase Modulation (SPM), Cross Phase Modulation (XPM) and Four Wave Mixing (FWM) [5].When a high-power optical signal is launched into a fiber, the linearity of the optical response is lost. One such nonlinear effect, which is due to the third-order electric susceptibility, is called the optical nonlinear effect.

The FWM occurs when light of two or more different wavelengths is launched into a fiber. Generally speaking FWM occurs when light of three different wavelengths is launched into a fiber, giving rise to a new wave (known as an idler), the wavelength of which does not coincide with any of the others.FWM is a kind of optical parametric oscillation [6].In other words, the FWM has been widely studied with various kinds of optical fibers. In particular, FWM has been investigated as a technique of realizing wavelength tunable light sources, and as a means of measuring fiber properties, such as the third order nonlinearity in a fiber [7]. In this paper, we proposed the new technique to suppress the FWM in SACOCDMA systems using the RD and the MD codes. This technique based on adding idle code at the sideband of the code construction to generate the virtual FWM power at the sideband of the signal, and then by subtracting these virtual FWM power from the original FWM power in the system and filtering out the data part at the channel.

\section{FOUR WAVE MiXING}

The origin of FWM process lies in the nonlinear response of bound electrons of a material to an applied optical field. In fact, the polarization induced in the medium contains not only linear terms but also the nonlinear terms. The magnitude of these terms is governed by the nonlinear susceptibilities of different orders.

The FWM process originates from third order nonlinear susceptibility ( $\left.\chi^{3}\right)$. If three optical fields with carrier frequencies $\mathrm{f1}, \mathrm{f} 2$ and $\mathrm{f} 3$, propagate inside the fiber simultaneously, $\left(\chi^{3}\right)$ generates a fourth field with frequency f4, which is related to other frequencies by a relation, $\mathrm{f} 4=\mathrm{f} 1 \pm \mathrm{f} 2 \pm$ 
f3. In quantum-mechanical context, FWM occurs when photons from one or more waves are annihilated and new photons are created at different frequencies such that net energy and momentum are conserved during the interaction. The effect of FWM on optical transmission is signal-to-noise degradation and cross-talk. As the signal input power of $\mathrm{f} 1, \mathrm{f} 2$, and $\mathrm{f} 3$ increases, or as the channel spacing decreases, the FWM mixing output increases [8, 9]. Figure (1), (2), and (3), explain the FWM power in different, input power, channel spacing, and fiber length respectively.

These results show that when power per channel is increased the spurious power increase, too. The power of the FWM produced is found to be inversely proportional to the square of the channel spacing, when all channels have the same input power. Furthermore, the FWM effects increase exponentially as the level of the optical power from the signal sources is increased, as shown in the figure (1). Based on results presented, it is clear that when the channel spacing is smaller, then the FWM effect becomes more significant due to the phase matching, as shown in Figure (2). In addition, the if the fiber length increases the FWM power decrease as shown in the figure (3). The equations below explain the FWM power works [10]:

$$
\Delta k=\left(\frac{2 \pi \lambda^{2}}{c}\right) \times \Delta f^{2}\left[D c+\frac{\lambda^{2}}{2 c} \times 2 \Delta f \times S D\right]
$$

Phase mismatches equation

Where SD $\rightarrow$ Slope Dispersion

Dc $\rightarrow$ Chromatic Dispersion

$\Delta \mathrm{f} \rightarrow$ Channel Spacing

$\lambda \rightarrow$ Wavelength

$\eta=\frac{\alpha^{2}}{\alpha^{2}+\Delta k^{2}}\left\{1+\frac{4 \exp (-\alpha L) \sin ^{2}\left(\frac{\Delta k L}{2}\right)}{[1-\exp (-\alpha L)]^{2}}\right\}$

FWM efficiency equation

$\alpha \rightarrow$ Attenuation

$\mathrm{L} \rightarrow$ Fiber Length

$P_{F W M}=\frac{1024 \pi^{6}}{n^{4} \lambda^{2} c^{2}}\left(\frac{d \times x_{1111} \times L e f f}{A e f f}\right)^{2} P_{\text {in }} \times \exp (-\alpha L) \times \eta$

$\mathrm{n} \rightarrow$ refractive index

Leff $\rightarrow$ effective Length

Aeff $\rightarrow$ effective Area

$\mathrm{X}_{1111} \rightarrow$ third order susceptibility.

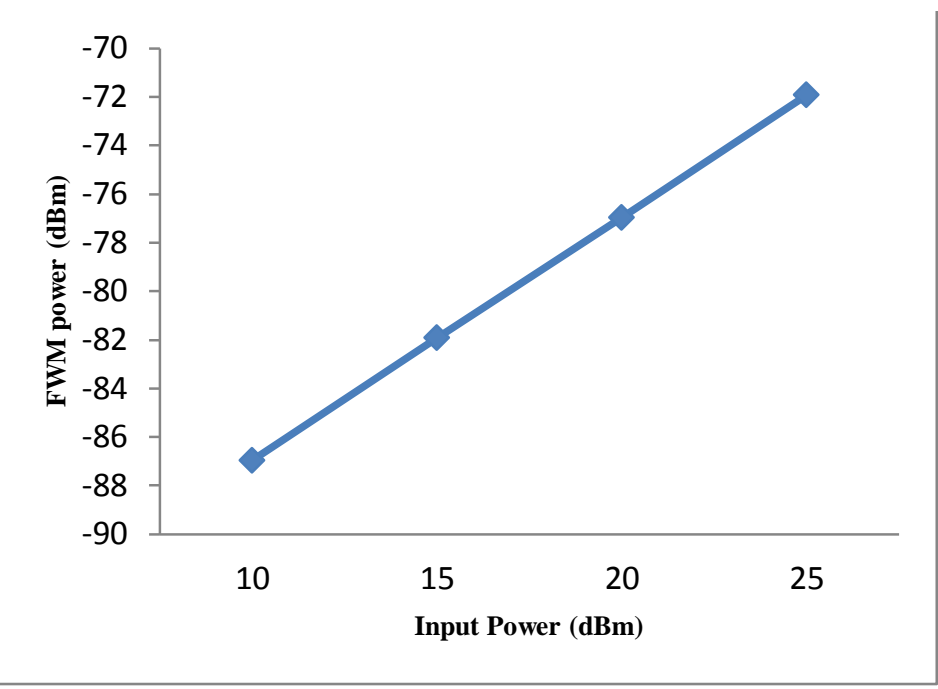

Fig.1. the FWM power versus Input Power

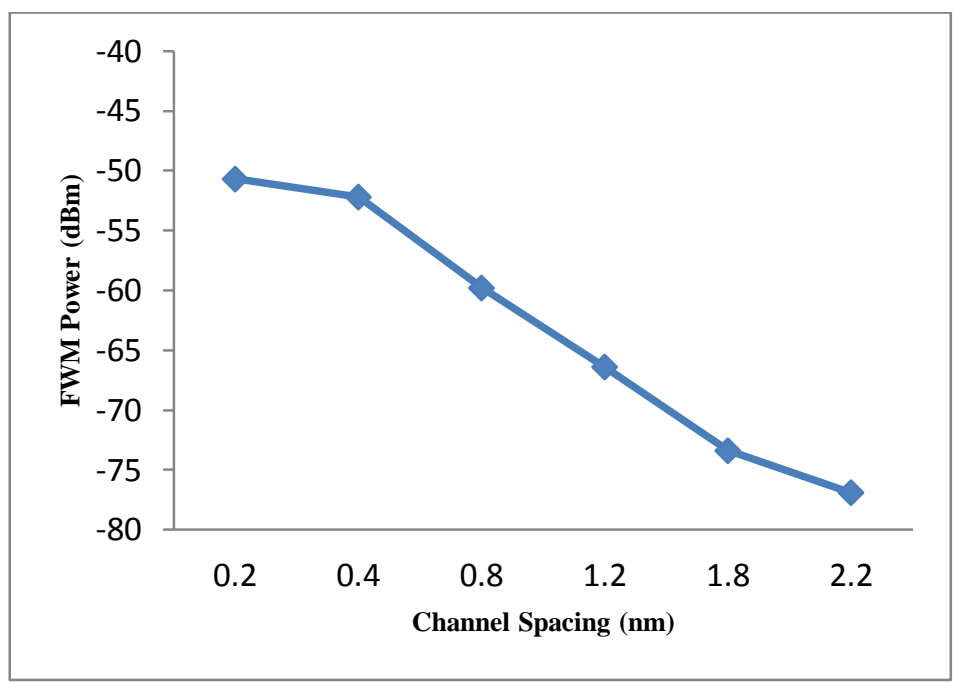

Fig.2. the FWM power versus Channel Spacing

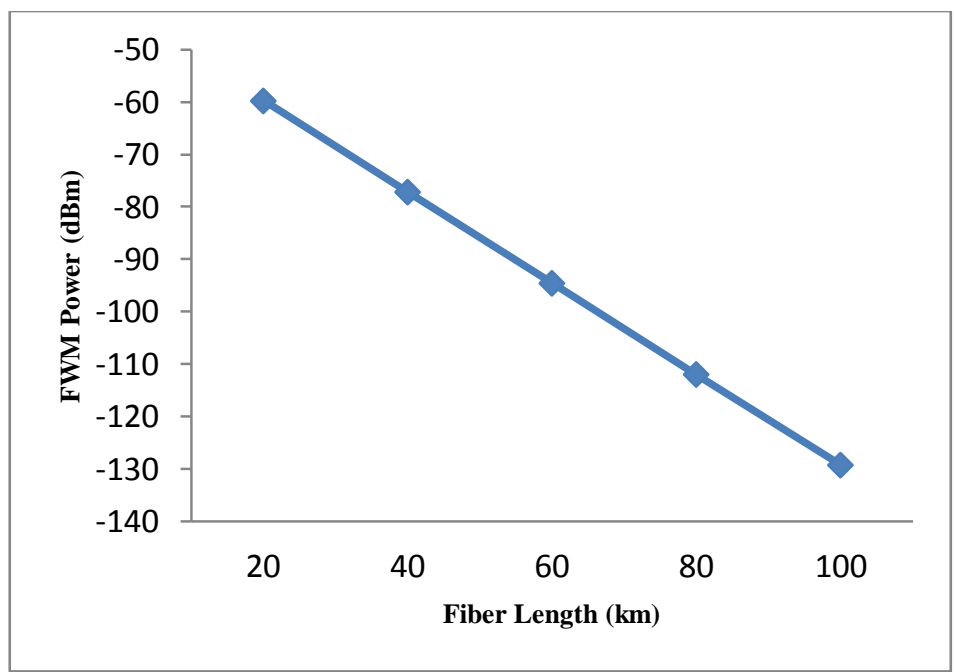

Fig.3. the FWM power versus Fiber Length 


\section{ThE NEW TECHNIQUE FOR SUPPRESSION THE FWM}

The FWM occurs when two or more frequencies of light propagate through an optical fiber together. Providing a condition known as phase matching is satisfied, light is generated at new frequencies using optical power from the original signals. Generation of light through four-wave mixing has serious implications for the rapidly expanding telecommunications field [9]. The generation of new frequencies from two or three input signals is shown in figure (4) below.

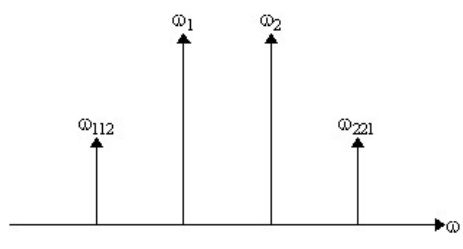

a)

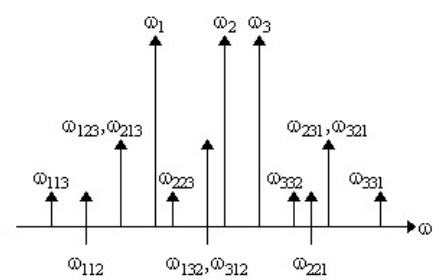

b)
Fig.4. the additional frequencies generated through in the partial degenerated (a) and non degenerated (b), the equation will be $w i j k=w i+w j-w k$ [11].

Consequently, in the SAC-OCDMA we generate the virtual FWM power at the sideband of the system design using the idle code (it will be explain in the next sections), and then subtracting the virtual FWM from the original FWM power in the system and filtered the data part in the channel using filters. This technique applied for SAC-OCDMA codes, the RD code and the MD code. Moreover, in terms of cost, the reported technique is considered a cost-effective as the LED light source is used to generate the sideband codes.

\section{SAC-OCDMA CODES}

\section{A. The RD Codes before and after Using the Technique}

The RD code is one of the important codes in the SAC OCDMA system. We will explain in this code according to the properties and the code construction that we used it in our simulation design. We suppress the FWM power in this code approximately $30 \mathrm{dBm}$ after using our technique. We denote a code by $(N, W, \lambda)$, where $N$ is the code length, $W$ is the code weight, and $\lambda$ is in-phase cross correlation. When $\lambda=1$, it is considered that the code possess ideal in phase cross correlation. The design of this new code can be performed by dividing the code sequence into two groups, which are code segment and data segment.

- Data segment: let the elements in this group contain only one " 1 " to keep cross correlation zero at data level $(\lambda=0)$. This property is represented by the matrix $(K x K)$ where $\mathrm{K}$ will represent number of users. These matrices have binary coefficientand a basic Zero cross code $(W=1)$ is defined as [Y1]. For example, three users $(K=3), y(K \times K)$ can be expressed as:

$$
[Y 1]=\left|\begin{array}{lll}
0 & 0 & 1 \\
0 & 1 & 0 \\
1 & 0 & 0
\end{array}\right|
$$

Where [Y1] consists of $(K \times K)$ identity matrices. Notice, for the above expression the cross correlation between any two rows is always zero. code segment: the representation of this matrix can be expressed as follows for $W=4$ :

$$
\text { [Y2] }=\left|\begin{array}{lllll}
1 & 1 & 0 & 1 & 0 \\
0 & 1 & 1 & 0 & 1 \\
1 & 0 & 1 & 1 & 0
\end{array}\right|
$$

Where [Y2] consists of two parts -weight matrix part and basics matrix part. Basic part [B] can be expressed as:

$$
[B]=\left|\begin{array}{lll}
1 & 1 & 0 \\
0 & 1 & 1 \\
1 & 0 & 1
\end{array}\right|
$$

And weight part called M matrix can be expressed as:

$$
[M]=\left|\begin{array}{ll}
1 & 0 \\
0 & 1 \\
1 & 0
\end{array}\right|
$$

Which is responsible for increasing number of weights. Let $i=(W-3)$ and $[\mathrm{Mi}]$ :

$$
[\mathrm{Mi}]=\left|\begin{array}{ll}
1 & 0 \\
0 & 1 \\
1 & 0
\end{array}\right|
$$

Where $\mathrm{i}$ represent number of Mi matrix on matrix $[\mathrm{M}]$, given by:

$$
\mathrm{M}=<\mathrm{M} 1|\mathrm{M} 2| \mathrm{M} 3 \ldots . . \mathrm{Mi}>
$$

For example, if $\mathrm{W}=5$, from Equation (4),

$$
[M]=\left|\begin{array}{llll}
1 & 0 & 1 & 0 \\
0 & 1 & 0 & 1 \\
1 & 0 & 1 & 0
\end{array}\right|
$$$$
\mathrm{i}=2 \text {, so that } \mathrm{M}=<\mathrm{M} 1 \mid \mathrm{M} 2>
$$

Notice that to increase the number of users simultaneously with the increase of code word length we can just repeat each row on both Matrixes $[\mathrm{M}]$ and $[\mathrm{B}]$. For Kth user matrix [M] and $[\mathrm{B}]$ can be expressed as: 


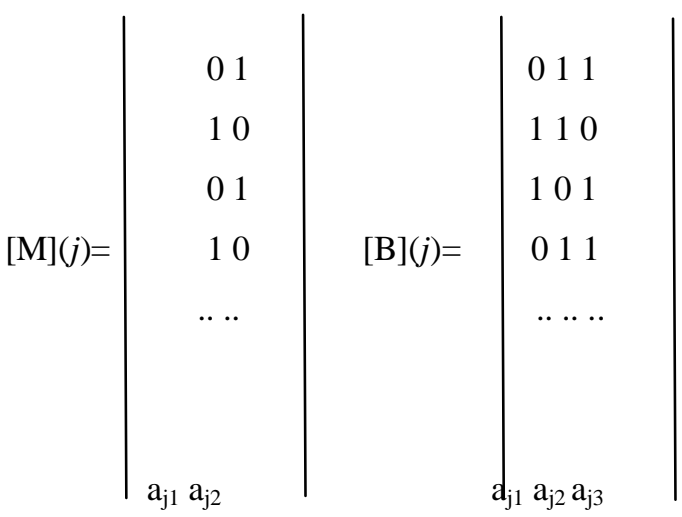

Where $\mathrm{j}$ represents the value for $K$ th user $(j=1,2 \ldots K)$, and the value of aj is either zero or one. The weights for code part for both matrix [M], [B] are equal to $W-1$, so the total combination of code is represented as $(K \times N)$ where $K=3, N=8$, as given by $[\mathrm{Z}],[\mathrm{Z}]=[\mathrm{Y} 1 \mid \mathrm{Y} 2]$. Notice that we will use $[\mathrm{M}]$ and [B] in other code as the code part to stimulate the FWM power

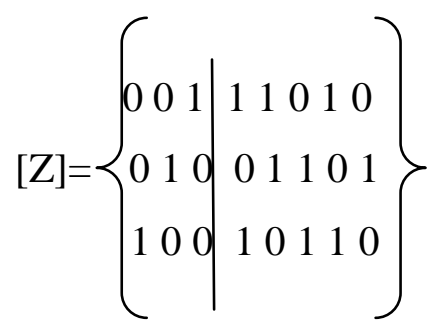

From the above basic matrix $Z$, determine the number of users $(K)$ and the code length $(N)$, as given by $(K \times N)$ matrix. Notice that the code weight of each row is equal to 4 , and the relation between $\mathrm{N}$ and $\mathrm{K}$ for this case $(W=4)$ can be expressed as:

$$
N=K+5
$$

As a result we can find that for $\mathrm{W}=5,6$, and 7 code, word length $\mathrm{N}$ can be expressed as $\mathrm{K}+7, \mathrm{~K}+9$ and $\mathrm{K}+11$ respectively. As a result the general equation describing number of users $\mathrm{K}$, code length $\mathrm{N}$ and code weight $\mathrm{W}$ is given as [12]:

$$
N=K+2 W-3
$$

And finally, after using the technique there is no change at this equations comparing with other codes (as will see in the MD code because the RD code is already using the code part as his construction. However, The RD code construction will change after using the technique as shown below:

$$
[\mathrm{RDx}]=[\mathrm{M}]|[\mathrm{Y} 1]|[\mathrm{B}]
$$

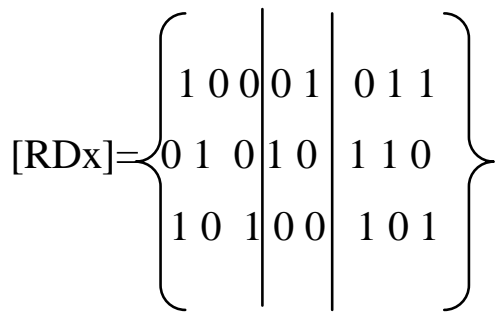

This section will be divided into two parts. Firstly, explaining the code construction of the MD code before using the technique. Secondly, explaining the code construction after using the technique. This code is also following by parameters $(\mathrm{N}, \mathrm{W}, \lambda \mathrm{c})$ that explained at the $\mathrm{RD}$ code in the previous section. This code has zero cross correlation, in linear algebra, the identity matrix or unit matrix of size $\mathrm{N}$ is the N-by-N square matrix with ones on the main diagonal and zeros elsewhere. It is denoted by IN, or simply by I if the size is immaterial or can be trivially determined by the context. From the MD matrix design in [13] the rows determine the number of users $(K)$. Notice that the association between code weight $(W)$, code length $(N)$ and number of subscribers $(K)$ can be expressed as:

$$
N=K \times W
$$

The total MD code sequence where $K=3, W=2$, and $N=6$ will be:

$$
\mathrm{MD}=\left|\begin{array}{llllll}
1 & 0 & 0 & 1 & 0 & 0 \\
0 & 1 & 0 & 0 & 1 & 0 \\
0 & 0 & 1 & 0 & 0 & 1
\end{array}\right|
$$

The MD code design depicts that changing matrices element in the same diagonal part will result in a constant property of zero cross correlation, and it is constructed with zero cross correlation properties. The MD code presents more flexibility in choosing the $\mathrm{W}, \mathrm{K}$ parameters and with a simple design to supply a large number of users [13]. After using the technique, the code construction of the MD will be change as we will explain below:

In this part, the matrix $[\mathrm{M}]$ will use at the sidebands. Consequently, the MD code construction after using the MD code will be:

$$
\begin{aligned}
& {[\mathrm{M} 1]=\left|\begin{array}{ll}
1 & 0 \\
0 & 1 \\
1 & 0
\end{array}\right|} \\
& {[\mathrm{MDx}]=[\mathrm{M} 1]|[\mathrm{MDx}]|[\mathrm{M} 1]}
\end{aligned}
$$

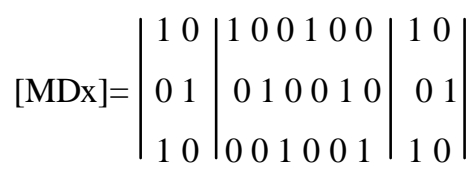

From the above basic matrices, the rows determine the number of users $(K)$. Notice that the association between code weight $(W)$, code length $(N)$ and number of subscribers $(K)$ can be expressed as:

$$
N=K \times W+4
$$

\section{Results AND Discussion}

\section{A. The FWM effects in the RD Code before and after using the technique}

\section{B. The MD Code before and after Using the Technique}


Figure 5 illustrate the FWM effects in the RD code before and after using the technique, the figure shows the FWM power versus the fiber length, the FWM power decreased after using the technique approximately $30 \mathrm{dBm}$. In addition, both figures ( $6 \mathrm{a}$, and $\mathrm{b}$ ), show the spectrum signal of the system before and after using the technique respectively and the FWM power signal shown at the sideband. It clearly shows that the FWM power suppress after using the technique. For example, at 40 $\mathrm{km}$ fiber length the FWM power values before and after using the technique is approximately $-55 \mathrm{dBm}$ and $-90 \mathrm{dBm}$ respectively.

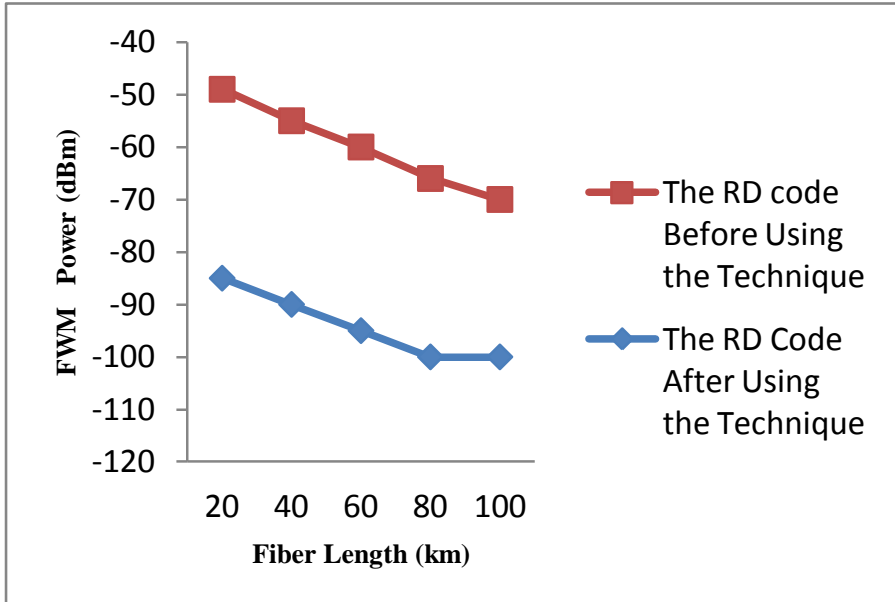

Fig.5. The FWM Power vs Fiber Length
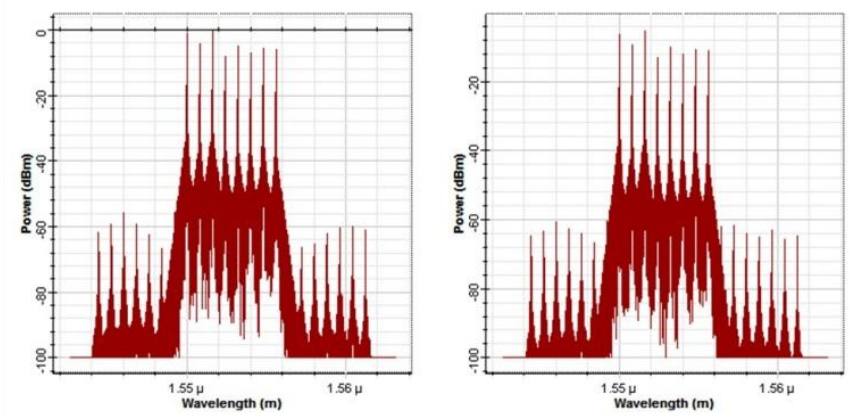

Fig.6. a) Spectrum of the FWM power at $40 \mathrm{~km}$ and $60 \mathrm{~km}$ respectively before using the technique
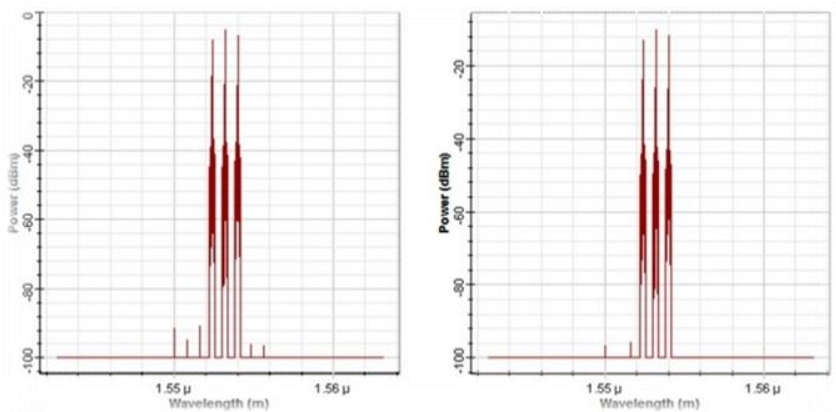

Fig.6. b) Spectrum of the FWM power at $40 \mathrm{~km}$ and $60 \mathrm{~km}$ respectively after using the technique

\section{$B$. The BER performance of the RD code before and after Using the Technique}

Figure (7) shows the BER versus fiber length before and after using the technique, the BER performance get better after using the technique. The data rate value of this system is 622 Mbps at input power $-10 \mathrm{dBm}$.

A longer length of fiber has a higher insertion loss, thus a smaller output power. In fact, when the fiber length decreases, the data rate should increase to recover a similar degradation of the signal form. Thus, in order to design and optimize link parameters, the maximum fiber length should be defined as short as possible, to obtain a high data rate and to achieve a desired system performance. In this system, the technique gives good performance when the fiber length is short as shown in figure (7). The performance of the system was characterized by referring to the BER and eye pattern.

The eye pattern for the RD code system is shown in figures 8 (a, and b), before and after using the technique at $35 \mathrm{~km}$ fiber length. The eye diagram gives a quick examination of the quality of the optical signal; it's clearly depicted that after using the technique, the code system gives a better performance, having a large eye opening. The vertical distance between the top of the eye opening and the maximum signal level gives the degree of distortion.

The more the eye closes, the more difficult it is to distinguish between $1 \mathrm{~s}$ and $0 \mathrm{~s}$ in the signal. Hence in our results in the data rate $622 \mathrm{Mbps}$ the BER at for the code before and after using the technique will be equal to $4.18 \times 10^{-23}$, and $1.6 \times 10^{-13}$ at $35 \mathrm{~km}$ and $40 \mathrm{~km}$ fiber length respectively before using the technique, after using the technique the BER values is $3.69 \times 10^{-28}, 4.05 \times 10^{-19}$ at $35 \mathrm{~km}$ and $40 \mathrm{~km}$ respectively.

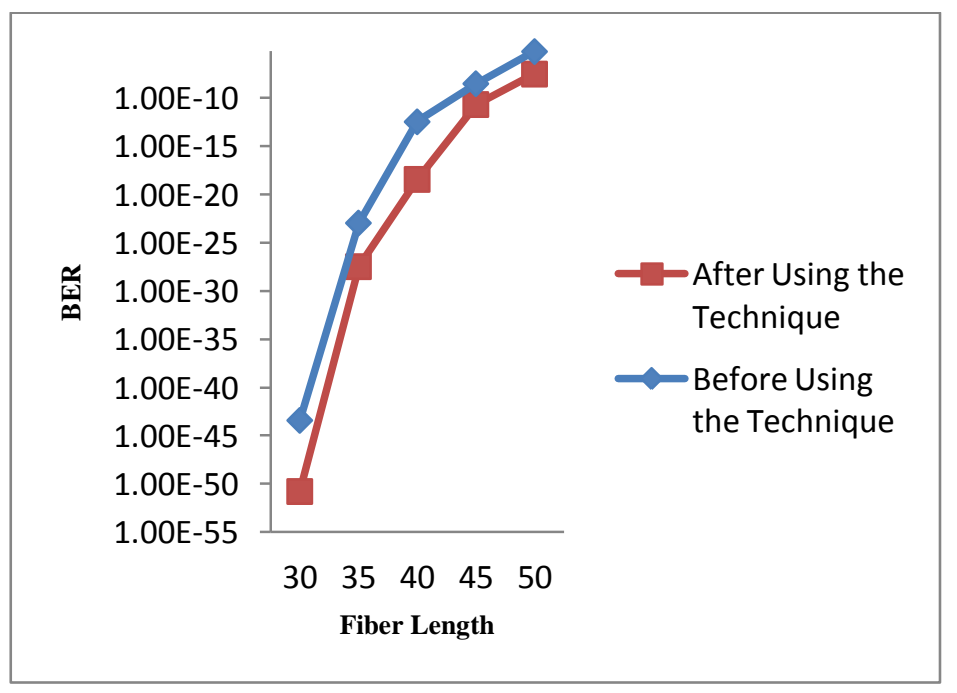

Fig.7. Variation of BER as a Function of fiber length 


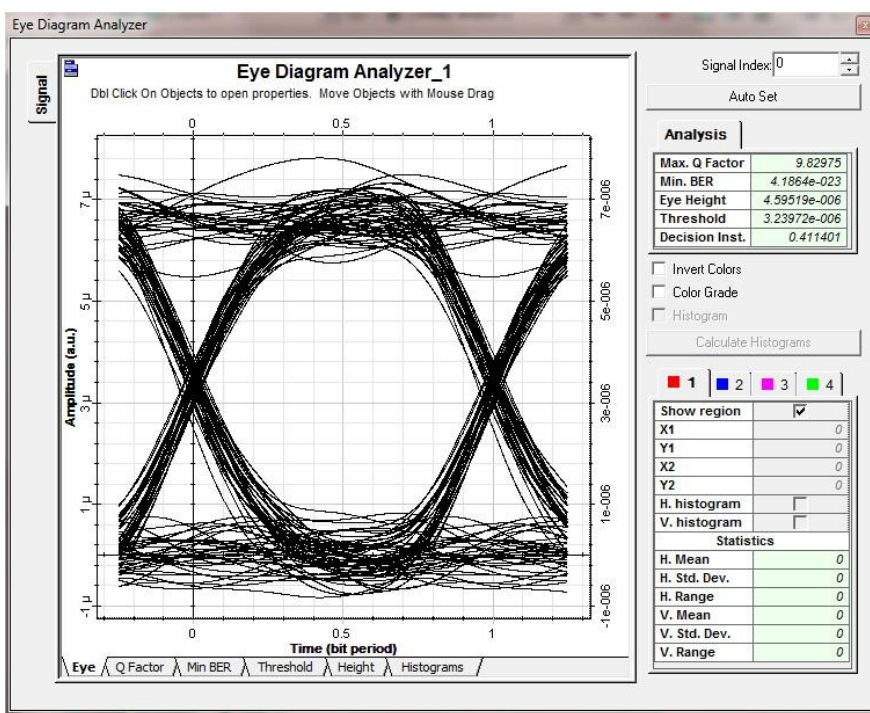

Fig.8. a) Eye diagram of the $\mathrm{RD}$ code at $35 \mathrm{~km}$ fiber length before using the technique

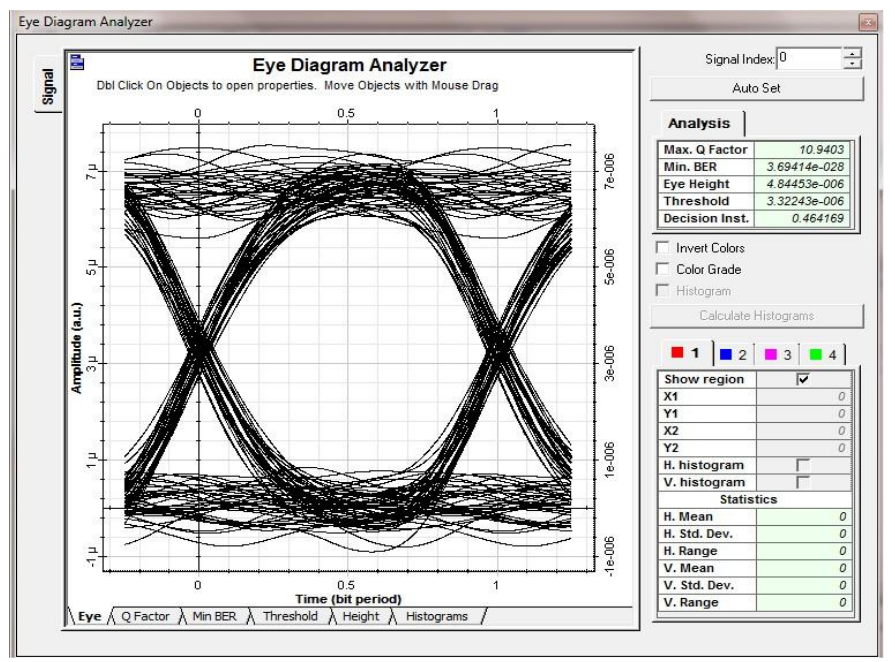

Fig.8. b) Eye diagram of the RD code at $35 \mathrm{~km}$ fiber length after using the technique

\section{The FWM effects in the RD Code before and after using the technique}

In the following process, the fiber length was varied from $20 \mathrm{~km}$ to $80 \mathrm{~km}$, it is clear that if the fiber length is lower effect of the FWM becomes very severe. From the results in figures (9), and (10 a, and b), the FWM power suppress after using the technique approximately $20 \mathrm{dBm}$. For example, at $40 \mathrm{~km}$ the FWM power before using the technique is approximately -60 $\mathrm{dBm}$ and after using the technique the FWM power value became $-80 \mathrm{dBm}$. Figures $10 \mathrm{a}$ and $\mathrm{b}$ show the spectrum of the MD code before and after using technique at $40 \mathrm{~km}$ and $60 \mathrm{~km}$ fiber length respectively.

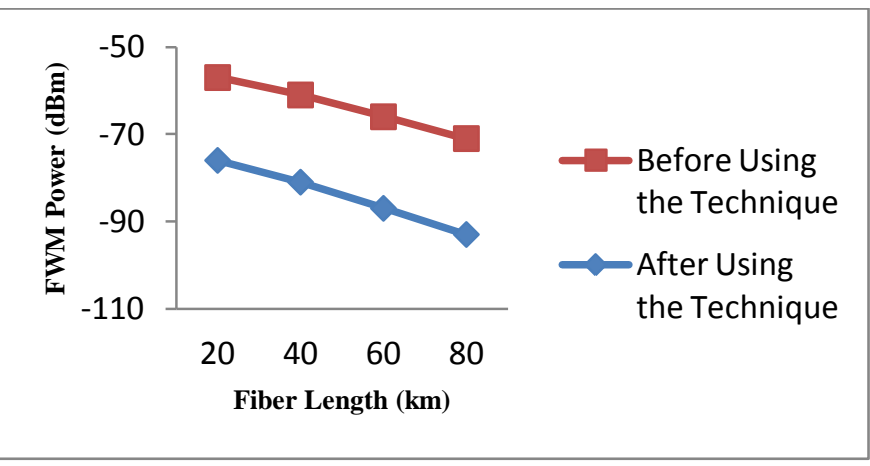

Fig.9. The FWM power vs fiber length
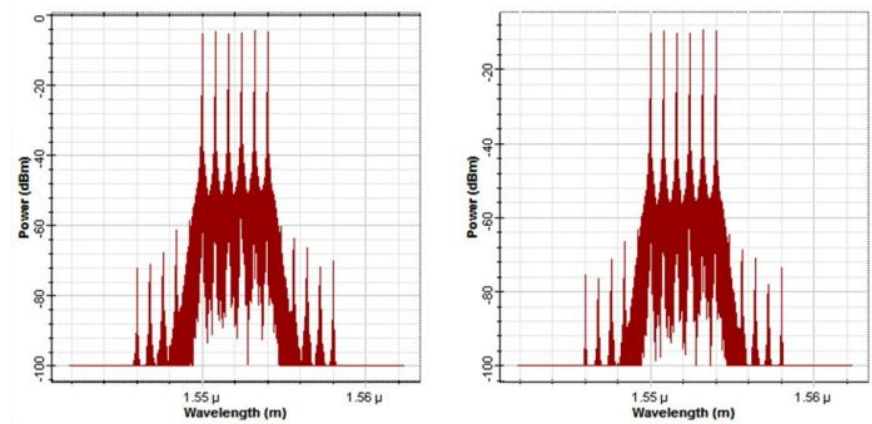

Fig.10. a) Spectrum of the FWM power at $40 \mathrm{~km}$ and $60 \mathrm{~km}$ respectively before using the technique
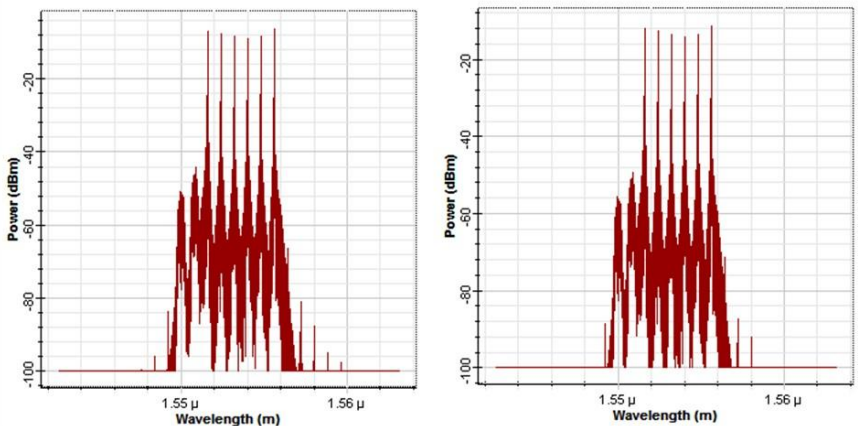

Fig.10. b) Spectrum of the FWM power at $40 \mathrm{~km}$ and $60 \mathrm{~km}$ respectively after using the technique

A. The BER performance of the MD code before and after Using the Technique

Figure 11 shows the relation between the BER and the fiber length based on the MD code before and after using the technique. It clearly shows that after using the technique the performance of the BER get better comparing with the system before using the technique. For example at $35 \mathrm{~km}$ the BER values before and after using the technique is $1.20 \times 10^{-23}$ and $3.6 \times 10^{-28}$ respectively. Figures $12 \mathrm{a}$, and $\mathrm{b}$, illustrate the eye diagram of the MD code before and after using the technique. 


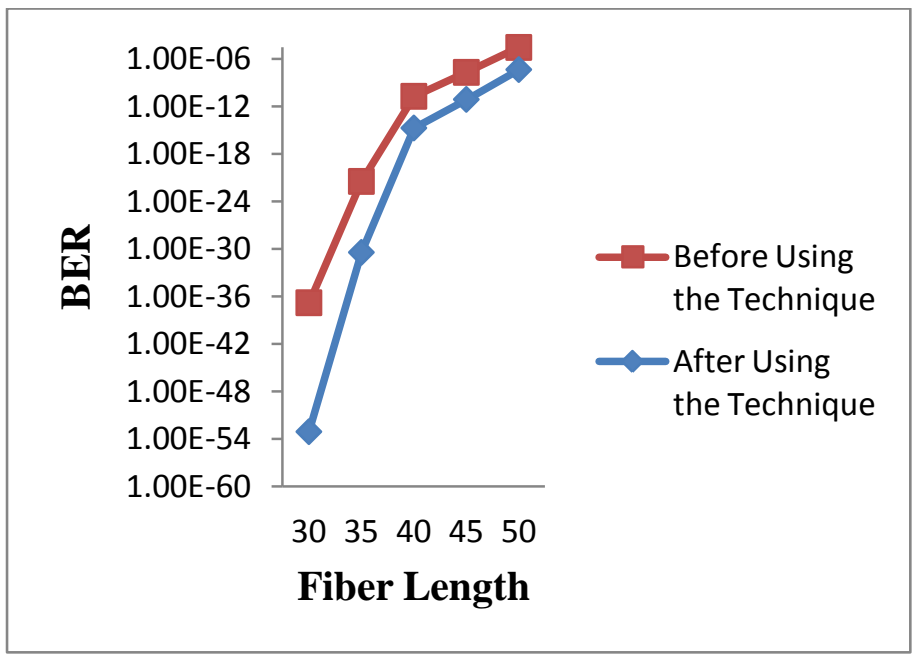

Fig.11. Variation of BER as a Function of fiber length

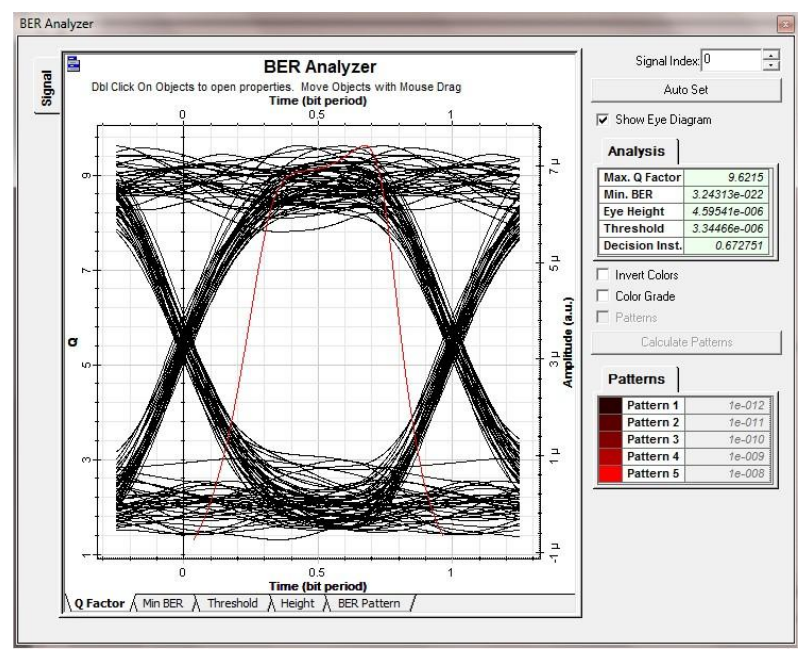

Fig.12. a) Eye diagram of the MD code at $35 \mathrm{~km}$ fiber length before using the technique

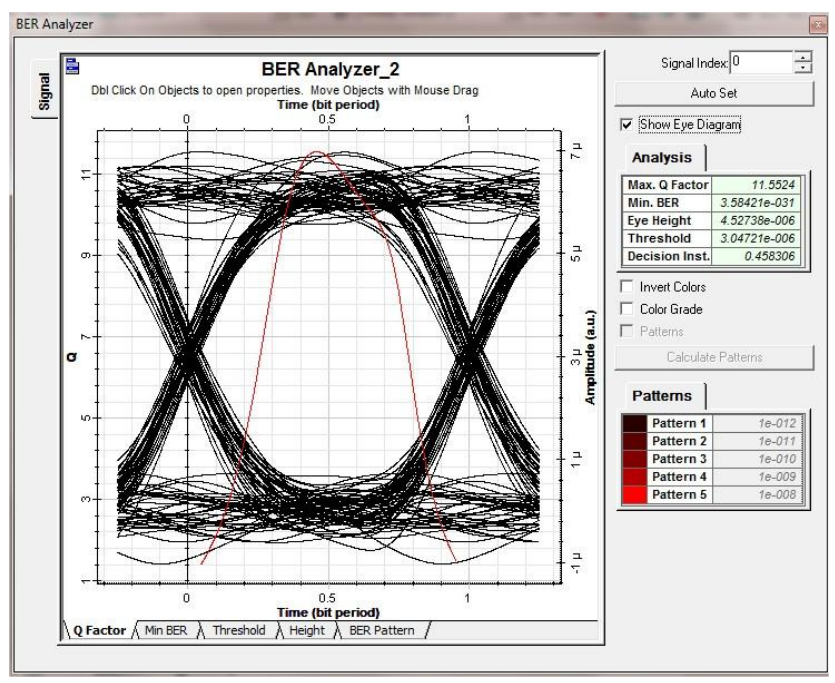

Fig.12. b) Eye diagram of the MD code at $35 \mathrm{~km}$ fiber length after using the technique

\section{CONCLUSION}

We have illustrated a new technique based on adding idle code at the sideband of the code construction to generate the virtual FWM power at the sideband of the signal, and then by subtracting these virtual FWM power from the original FWM power in the system and filtering the data part at the channel. This technique is applied for both SAC codes, the RD and the MD codes. The systems give better performance in BER after using the technique comparing with the systems before using the techniqe. In addition, the FWM power reducing in the MD code more than $20 \mathrm{dBm}$ and in the RD code more than 30 $\mathrm{dBm}$. For example, in the RD code the FWM power at $40 \mathrm{~km}$ fiber length and input power is $15 \mathrm{dBm}$ is approximately -55 $\mathrm{dBm}$ before using the technique, after using the technique the FWM power is approximately $-90 \mathrm{dBm}$. In other words, at the MD code the FWM power before using the technique is approximately $-61 \mathrm{dBm}$, the same parameters values, however after using our technique the value of the FWM power is approximately $-81 \mathrm{dBm}$. In adittion, for example at the BER values, the BER in the RD code at the input power $-10 \mathrm{dBm}$ and $35 \mathrm{~km}$ fiber length before using the technique is $1.6 \times 10^{-23}$ and after using the technique the value of BER will become $4.05 \times 10^{-28}$. In addition at the MD code the BER value before using the technique is $9.4 \times 10^{-22}$ and after using the technique the value of BER is $7.4 \times 10^{-31}$.

\section{REFERENCES}

[1] Abtin Keshavarzian, J. A. S. "Optical Orthogonal Code Acquisition in Fiber-Optic CDMA Systems via the Simple Serial-Search Method." IEEE Transactions on Communication Vol. 50, No. 3 (2002).

[2] Indu Bala, V. R. "Performance analysis of SAC based non-coherent optical CDMA system for OOC with variable data rates under noisy environment." Indian Journal of Science and Technology Vol.2 No. 8: 49-52 (2009)

[3] Harmandeep Singh, Dr. YADUVIR SINGH. "Performance Analysis of Optical CDMA using Fuzzy Logic Generator”. Department of Electrical and Instrumentation Engineering, THAPAR UNIVERSITY (2008).

[4] [4]. Fuad A. Hatim, F. N. H., Sahbudin Shaari. "Effects of Nonlinear Stimulated Brillouin Scattering on Performance Analysis of an Optical CDMA Transmission System." Journal of Optical Communications 30: 104-108(2009).

[5] Boyd, R. W, "Nonlinear Optics (2nd ed.)". USA: Elsevier Science (2003).

[6] Osamu Aso, M. T., Shu Namiki. "Four-Wave Mixing in Optical Fibers and Its Applications." Furukawa Review 19: 63-68 (2000)

[7] K.P. Lor, K. S. C.. "Theory of nondegenerate four-wave mixing in a birefringent optical fibre." Optics Communications 152: 26-30 (1998)

[8] Banerjee, P. ," Nonlinear Optics: Theory, Numerical Modeling, and applications". New York: Marcel Dekker, Inc. (2004).

[9] Singh, A., Sharma, A. K., \& Kamal, T. S, "Four-wave mixing analysis in WDM optical communication systems with higher-order dispersion". Optik - International Journal for Light and Electron Optics, 119(16), 788-792. (2008).

[10] S. P. Singh, N. S.. "Nonlinear Effects In Optical Fibers: Origin, Managment And Applications." Progress In Electromagnetics Research, PIER Vol. 73: 249-275 (2007).

[11] Billington, R. "A report on four-wave mixing in optical fibre and its metrological applications". R - Report. United Kingdom (1999). http://www.opengrey.eu/item/display/10068/697218

[12] Fadhil, H. A., Aljunid, S. A., \& Ahmad, R. B, "Performance of random diagonal code for OCDMA systems using new spectral direct detection technique". Optical Fiber Technology, 15(3), 283-289. (2009).

[13] Abd, T. H., S. A. Aljunid, et al. "Development of a new code family based on SAC-OCDMA system with large cardinality for OCDMA network." Optical Fiber Technology 17(4): 273-280. (2011). 